\title{
Exploiting Granular Electrical Energy Data and the Challenges a Utility Company Faces with an Ageing Asset Base
}

\author{
Alex D.L. Gray \\ Ioana Pisica \\ Brunel London - Englanc Brunel London-England \\ Gareth A. Taylor \\ Alex.Gray@brunel.ac.uk Ioana.Pisica@brunel.ac.uk \\ Brunel London - England \\ Louisa Whitehurst \\ Gareth.Taylor@brunel.ac.uk \\ Thames Water - England \\ Louisa.Whitehurst@thameswater.co.uk
}

\begin{abstract}
Within the UK water industry, electrical energy demand, efficiency and sustainability are primary factors when considering the construction and installation of new electrical process plant. This stems from the environmental gains made in reducing electrical power consumption, and the cost saving benefits realised across an electrical assets lifecycle. The average life of electrical assets also varies greatly, and with the speed at which new technology is being developed, they also quickly become superseded. Additionally, the electrical data you can extract from newer assets has become more granular, for example a motor control centre will now provide fieldbus power monitors per pump, rather than a single CT/VT monitor on the incomer. This provides the ability to pinpoint areas where further efficiencies can be achieved and/or were certain devices are failing.

Difficulties however, manifest when trying to understand how the older electrical assets of a process site are performing from an energy perspective. Further still. If these devices are not on the roadmap for replacement, then the potential for granular power data may not be realised for some time. On these sites, the site power is seen is a single unit of measure, in the MWh range, whereas it is now a requirement for device level readings as low as single figure $\mathrm{kWh}$. Therefore, in order to achieve accurate results from the older assets, power monitoring equipment requires retrofitting. This paper will investigate the challenges involved in installing a sub-metering system across an existing, aging electrical asset base, where monitoring was not engineered within the original design. Further to this, it will discuss the obstacles faced when transferring data from site through to corporate historians and provide recommendations for future work.
\end{abstract}

Index Terms - Sub-metering, Energy, SCADA, Control Systems, Data Lifecycles, Process Design, Water Utility, Regulators, Energy Investment Strategies, Automatic Meter Reading.

\section{INTRODUCTION}

$\mathrm{E}$ lectrical sub-metering is now an essential part of energy monitoring within large industrial and commercial establishments, offering advances in efficient building and asset-centric management [1]. For the larger consumers, it provides the ability to analyse lower level data for auditing purposes, which in turn can aid in targeted energy reduction and reduce the capital energy spend. [2]. By recording the sub-metered energy data, through data historians such as supervisory control and data acquisition (SCADA) systems, will give analysists the ability to trend the electrical consumption patterns at a more granular level and thus provide the tools to determine where potentially poor performing assets exist. This in turn can instigate reactive measures to be taken to either reduce the use of the particular asset, through reprioritisation (switch to the backup device), or by complete replacement of the failing device. When this granular data is presented to the end user, it highlights the amount of wasted energy and areas to target for achieving efficiency savings [2].

The electrical controls infrastructure throughout the Thames Water estate varies greatly, with some motor control centres (MCC's) being up to 35-40 years old. These panels have no energy monitoring systems installed; there could be a single current transformer (CT) that is installed on a single phase of an electrical starter, which provides an analogue current meter with a secondary scaled current of 0-5Amps. These $0-5 \mathrm{~A}$ analogue signals are not in a compatible format to be presented to a control and instrumentation (C\&I) device, such as a programmable logic controller (PLC), and therefore, the signal cannot be transferred to the SCADA systems. Conversely, the more recent MCC's installed come ready equipped with intelligent power monitoring that utilise Fieldbus communication protocols and integrate directly to PLC's over networked control systems (NCS).

Across the 4000 Thames Water sites, the electricity is measured by Automatic Meter Reading (AMR) meters which log and track exactly how much electricity is being used by the business. Each AMR meter produces individual readings for every half-hour in the year, this equates to an average of 68 million half-hours of data per year for Thames Water, which in turn is used for billing and forecasting. [3]

The AMR meters are most often installed close to the point of common coupling (PCC), between the distribution network and the end users demand side. For the larger industrial processes, these AMR meters are generally located in onsite substations, which have high voltage incomers up to and including $33 \mathrm{kV}$, although the most common intake voltage is $11 \mathrm{kV}$.

On the very large sites, Thames Water own and maintain their own high voltage (HV) and medium voltage (MV) network systems, however, the AMR meter will still only 
exist at the PCC, regardless of the voltage rating. This is sufficient for capturing the entire sites half-hourly energy consumption and can be presented to the electric companies, and in some cases Thames Water. The data is transferred via low bandwidth technology that takes a digital pulse output from the AMR meter head and transmits it to the supplier for billing purposes. The pulse can be calibrated to equal defined unit measures of energy in $\mathrm{kWh}$ or MWh. Fig. 1 below is an example of an $\mathrm{HV} / \mathrm{LV}$ system within a large process plant. It has been scaled down for the purpose of this paper, but still shows the linkage from the Distribution Network Operator (DNO) substation trough to the endpoint devices, such as pumps and distribution boards. As can be seen from the diagram, the AMR energy metering is only present at the PCC.

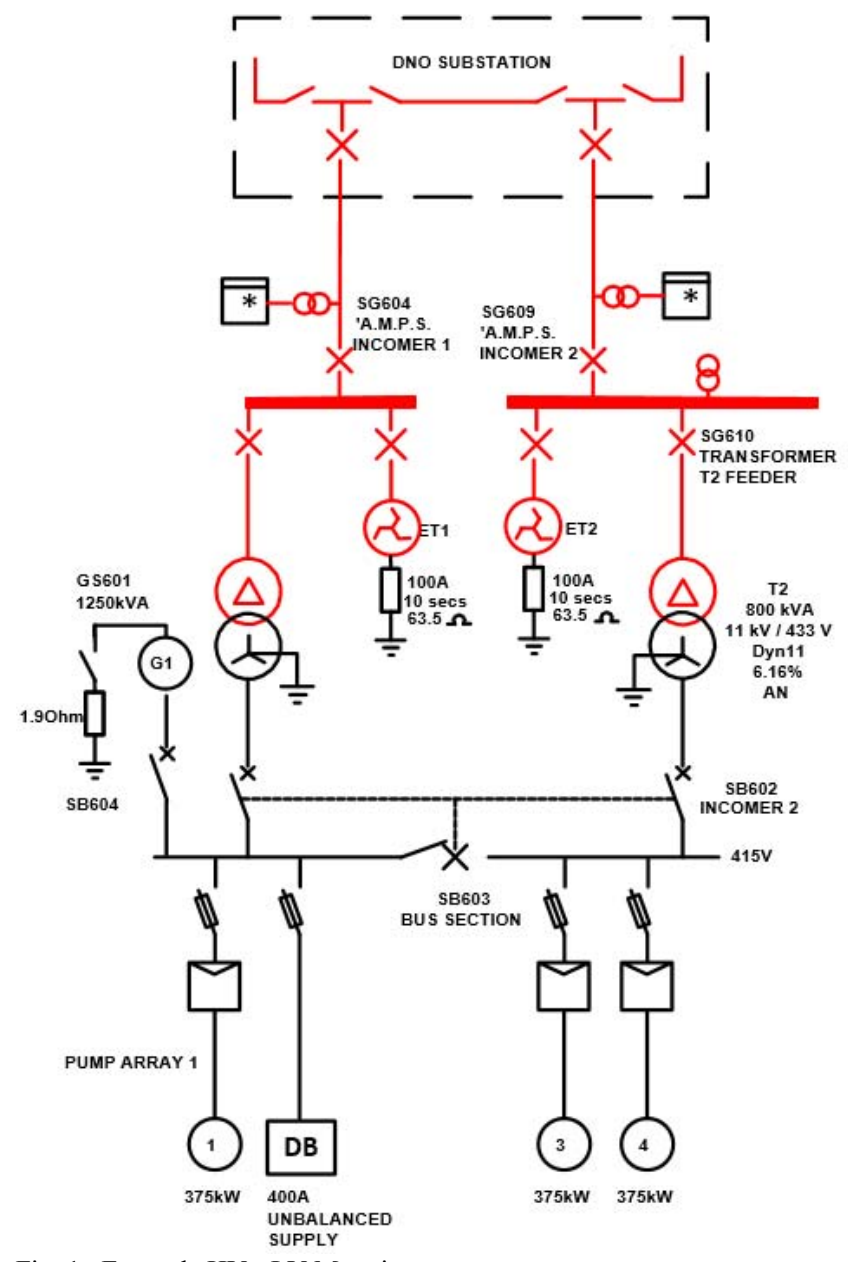

Fig. 1. Example HV - LV Metering

With rising energy prices and an increase in the volatility of the market, it is advantageous for Thames Water to be more in control of its energy consumption and management so that it may reduce operational expenditure, as well as prolonging asset life cycles [4].

Growing pressures from the regulators on the reduction of carbon footprint have contributed towards a shift in response from the water companies. Asset management periods (AMP's) are investment cycles, which are determined by the economic regulator of the water sector in England and Wales (OFWAT), via 5-yearly price reviews. These reviews cap price limits for the regulated water companies in England and Wales and set strategies for capital investment. For AMP5, which was the period between 2010 and 2015, these price limits allowed for a further $£ 22$ billion of capital expenditure. There was huge focus on renewable energy generation, with a target combined output of $300 \mathrm{GWh}$ across the $12 \mathrm{UK}$ water companies [5]. However, OFWAT did not believe it was necessary for the companies to put notified items or cost pass through mechanisms in place for guarding against future increases in energy costs because 1) changes in energy prices are a risk that Retail Prices Index (RPI) indexation partly mitigates, and 2) the base operating expenditure figures they took forward from 2008-09 into price limits already included energy costs at a level they thought was appropriate for many companies [5].

This led to Thames Water making a business decision to react to, and protect against these volatile energy prices, by investing in an energy sub-metering programme that looked to extract energy data from individual electrical assets, such as waste water aerators, large transfer pumps, borehole pumps, drives and any other device seen as high usage, and ultimately high potential for efficiency savings. The intention being, to present this data on the corporate SCADA systems and data historians, all with a view that poor performing devices could be identified, removed from service and/or replaced with an alternative (more efficient) device.

Challenges exist all the way through the data chain transfer; with the physical limitations presented by the legacy MCC's being more apparent at the meter installation stage. However, even after the signals are digitised, and presented to the local PLC/SCADA systems, there are further challenges surrounding configuration, offsite exportation, scaling and accuracy issues.

This paper will present these challenges, along with the ways in which some were mitigated against, the benefits realised following the access to granular energy data, and recommendations towards further enhancing the electrical estate and its energy monitoring.

Section II discusses the challenges and varied architectures within the Thames Water electrical estate, section III explores the 'Data-Flow' architecture with the full end-to-end cycle for energy meter to end user being discussed, section IV reviews current benefits being realised, with the conclusions presented in section $\mathrm{V}$.

\section{LEGACY ELECTRICAL ASSETS}

Upon project initiation, there were various electrical architectures that required understanding prior to the power monitoring and data transfer. Some required more invasive installation works than others, however, the following categories summarised the majority of systems presented.

1) High and Medium Voltage Feeders

The HV/MV systems comprised old power meters and/or needle meters on the panel fascia. These were supplied via current transformers (CT's) and voltage transformers (VT's). The meters had no usable outputs that could be integrated with the control system, therefore they required replacement. 


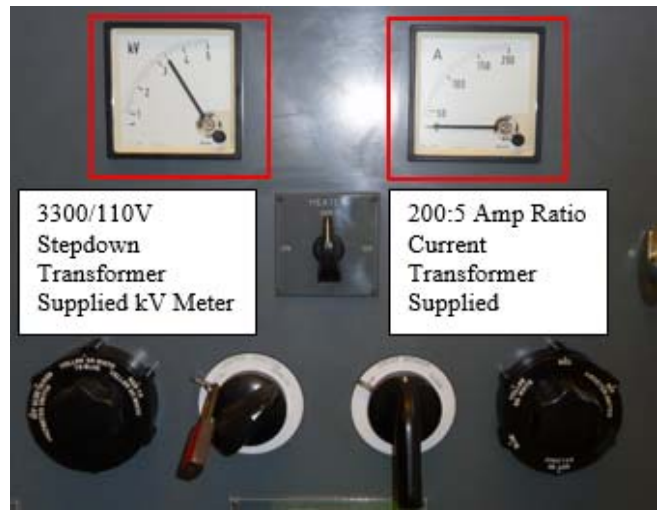

Fig. 2. 3.3kV Switchgear Section

Fig. 2 above shows an HV section of a large distribution main, which was one of the feeders that required monitoring. The critical nature of the downstream plant it supplied meant that shutdown times needed planning months in advance and that the duration could not exceed 30 minutes at a time. This put a large dependency on logistics planning, to ensure all engineering resource was lined up and able to complete their respective tasks without impedance.

2) Existing LV Power Meter Replaced - Discrete Probably the least invasive in terms of installation requirements, as there was an existing, non-compatible meter in place that just needed to be upgraded for a new unit. In most cases the CT's were already installed, and as the systems were LV, there was no need for a transformed voltage supply, as a $400 \mathrm{~V}$ reference was available.

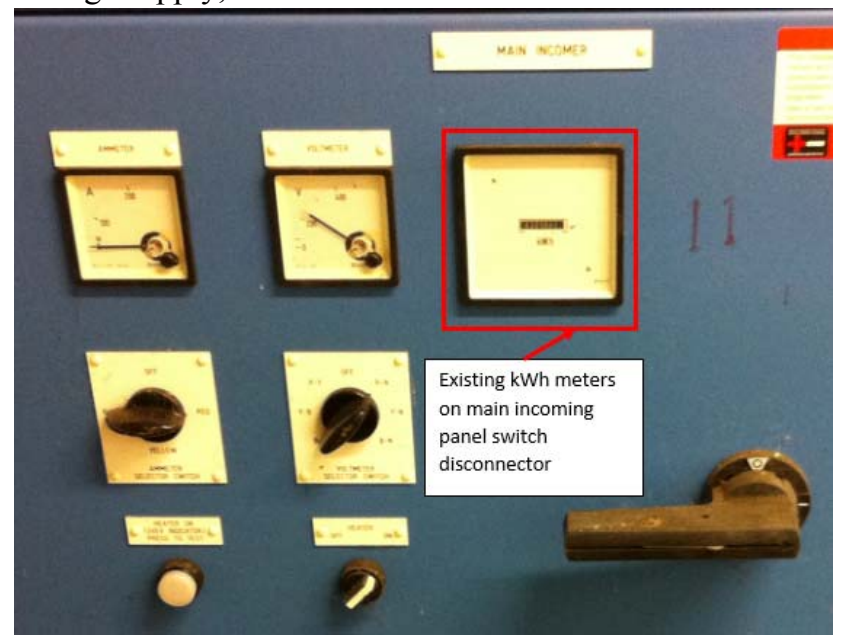

Fig. 3. 400V Switch Disconnector - with legacy kWh Meter

However, in some cases, when the section requiring installation also comprised an interlocked switch disconnector, as shown in Fig. 3, then full electrical isolations were required. Any downstream plant would therefore, need to be removed from service, impacting the associated processes. Sometimes it was not always possible to take these systems offline due to other dependencies, such as such as rainfall, consumer demand etc. By the very nature of the parameters that governed this, it would also mean that the installation engineers could be prevented from undertaking the work, on the day they arrived, without any forewarning. These preventions from work heavily impacted the companies commercially, through revisits and project prolongation costs.

3) Existing Power Meter Replaced - Fieldbus

On some of the more modern sites, there were existing power meters that comprised Fieldbus connections to the PLC/SCADA network. In these circumstances, all that was required was the recommissioning of the power instrument. However, in some cases, such as the 3300 ACM unit shown below in Fig. 4, the old RS-485 output would be connected to failed legacy PLC communications modules. Where the communications module in the PLC was still in service, the recommissioning of the signals was a relatively low impact task. However, due to the cost of replacement units being high, and the availability lead times in excess of 3 months, it was sometimes deemed more cost effective to replace the unit with a new sub-meter when the modules were failed.

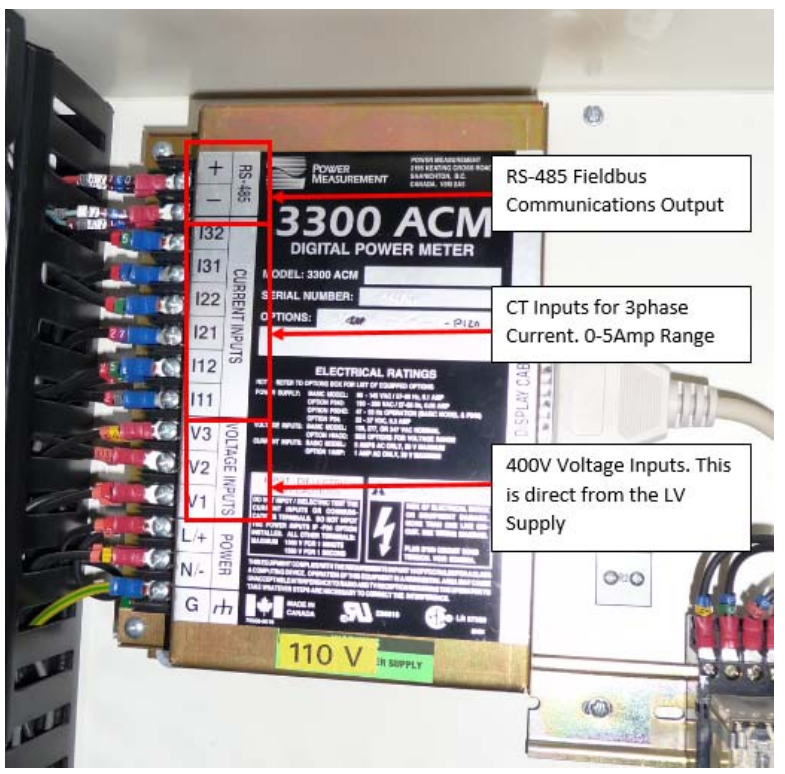

Fig. 4. Existing Fieldbus Power Monitor

4) Full Installation of new Power Meter

The most invasive of all sub-metering installations, therefore not permitted on any $\mathrm{HV}$ or MV system due to a risk level that was deemed unacceptable. For electrical compartments where no existing monitoring was available, the installation of all power monitoring equipment was required. The largest risk from this was the CT installation. Some of the older feeders had solid copper bus-bar systems and this meant wholesale reconfiguration was needed, which in turn meant longer outages on the target device. Operational restrictions would in most cases dictate that a return to service time of 15 minutes was in place, such that if the process environment changed and operations needed the device back in service, they could instruct this at any moment. This restriction meant the engineering team had to break the installation down into sub-components. The CT's were installed one phase at a time, which reduced the exposure of having all 3 phases disassembled during the works. The cabling that connected the CT secondary's back to the submeter were pre-loomed away from the panel and installed between the compartments as an additional sub-component task. Where this approach did increase the overall installation time, it was considered a mandatory method, as it was crucial for maintaining the 15 minute return to service.

\section{A. Power Meter and Electrical Configurations}

The sub-meter chosen was a Socomec Diris A40, and based on affordability and flexible connection to different LV system types, such as non-balanced loads, balanced loads, HV 
systems with transformed voltage references and limited CT availability. It also conforms with the European Measuring Instruments Directive (MID 2004/22/EC) [6]. If an energy meter conforms with MID, then, from the 30th October 2006, the UK government permitted it towards the use for any renewable obligation certificate schemes (ROCS) or feed in tariff schemes (FIT) [7].

Generally, for any LV distribution panel, which incorporates building services and/or unbalanced systems, the unbalanced 3 phase wiring configuration was required for ensuring the greatest accuracy. Fig. 5 below shows the wiring schematic for this particular configuration. It is a 3-phase 4wire connection and replicates the three Watt meter arrangement.

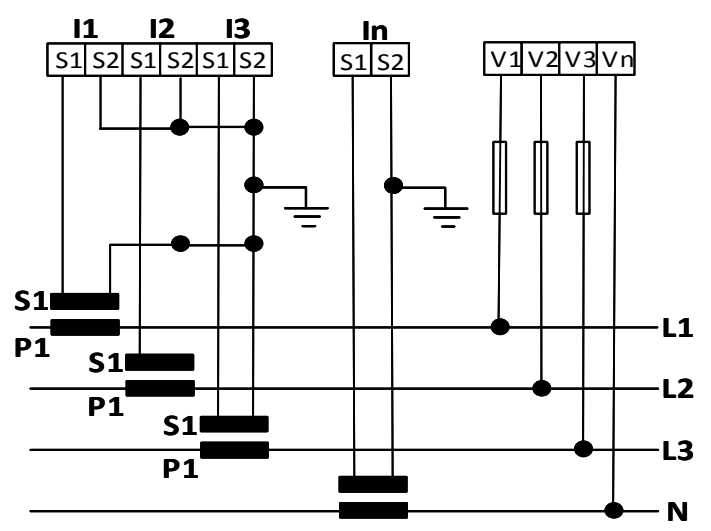

Fig. 5. Unbalanced 4-Wire 3 Phase Connection

Fig. 6 below therefore, depicts that for a 3-wire system, the sub-meter can be configured as a two Watt meter measurement device.

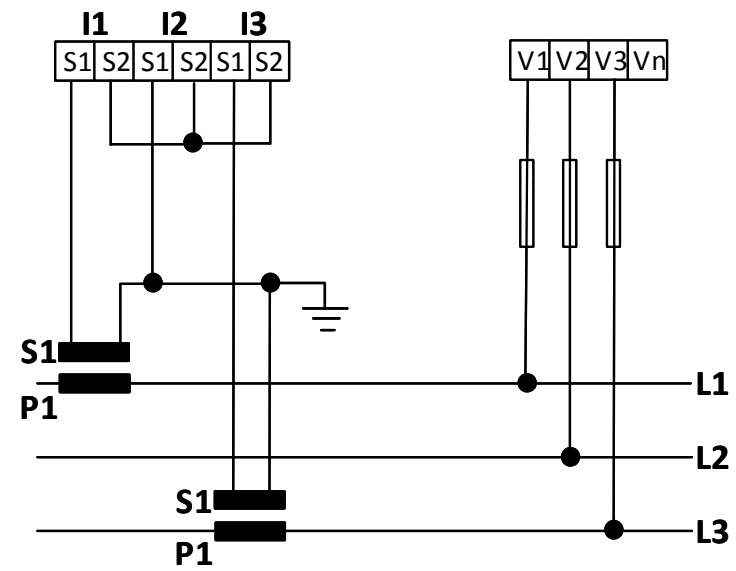

Fig. 6. Unbalanced 3-Wire 3 Phase Connection

Where full installations were required on any unbalanced load, the installation of the 4-wire, three Watt meter configuration was employed, i.e. 4 CT's installed. However, where CT's already existed on 2 of the 3 phases of the target device, then, to save on installation costs, the 3-wire, two Watt meter configuration could be used, because the unit could calculate the 2 nd and 3rd phase currents by vectoral summation. This did result in a $0.5 \%$ reduction in phase accuracy [9], but was deemed negligible in contrast to the risk and cost associated with installing additional CT's, and so was an acceptable accuracy burden.

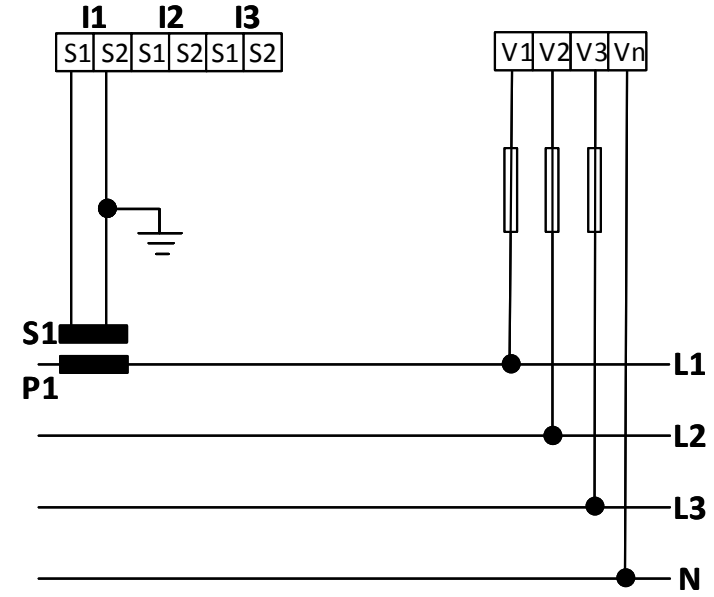

Fig. 7. Balanced 3or 4 wire 3 Phase Connection

For most pumps, motors and 3-phase balanced loads, the most cost-effective way to obtain the power measurement was through the one Watt meter approach, as shown in Fig. 7. As all phases must be balanced for these devices, there is no need for vectoral summation as the line current in the measured phase must equal the line current in the two remaining unmeasured phases.

\section{B. Electrical Safety and Risks}

Even after the metering cubicles isolation, with the larger MCC's and/or electrical distribution panels, there remained a potential for live parts to still exist within the metering cubicles. This was due to the CT's and VT's being installed on the system prior to the main isolation of the respective panel. As a priority, the electrical team's main challenge was to completely remove this potential risk from live parts; however, in some cases this was not always possible due to operational restrictions. There were also certain issues with the integrity of the shrouding systems that provided a barrier between the main switchgear disconnector load terminals and the associated supply terminals. On the older panels, the shrouding, which was constructed from an acrylic type material, had become brittle and could not be trusted to provide a suitable barrier that could withstand impact from external influence.

The electrical team had to assess the cubicle feed system to ascertain where the energy source was entering. If the source was still live after local isolation (the supply side of the isolator), then they had to further assess whether or not additional upstream isolations were possible, so as to completely eliminate all electrical risk from the cubicle.

Where further upstream isolations were not feasible, they had to carry out a non-contact, assessment of the shrouding system (i.e. no hands or tools to touch anything within) that protected the live terminals. If the source of supply entered the panel at the top, and the competent person deemed the shrouding integrity to be suitable against accidental ingress from tool or human contact, then work was permitted to commence. If the source of supply entered from a lower point than the area being worked in then the electrical competent person was not permitted to work until an additional Safe System of Work (SSOW) had been completed. In most cases, the source of supply entered from the bottom, and therefore there were multiple additional delays in determining further SSOW's, which in most cases required upstream isolations. 


\section{DATA-FLOW ARCHITECTURE}

By connecting AMR meters to the offsite historian system completes the operational chain from Substation to customer [10] and therefore increases visibility.

As part of the sub-metering scheme, there was a requirement to store process performance data, both in the short and long term. For the short-term requirements, it was deemed that control room operators needed to trend a

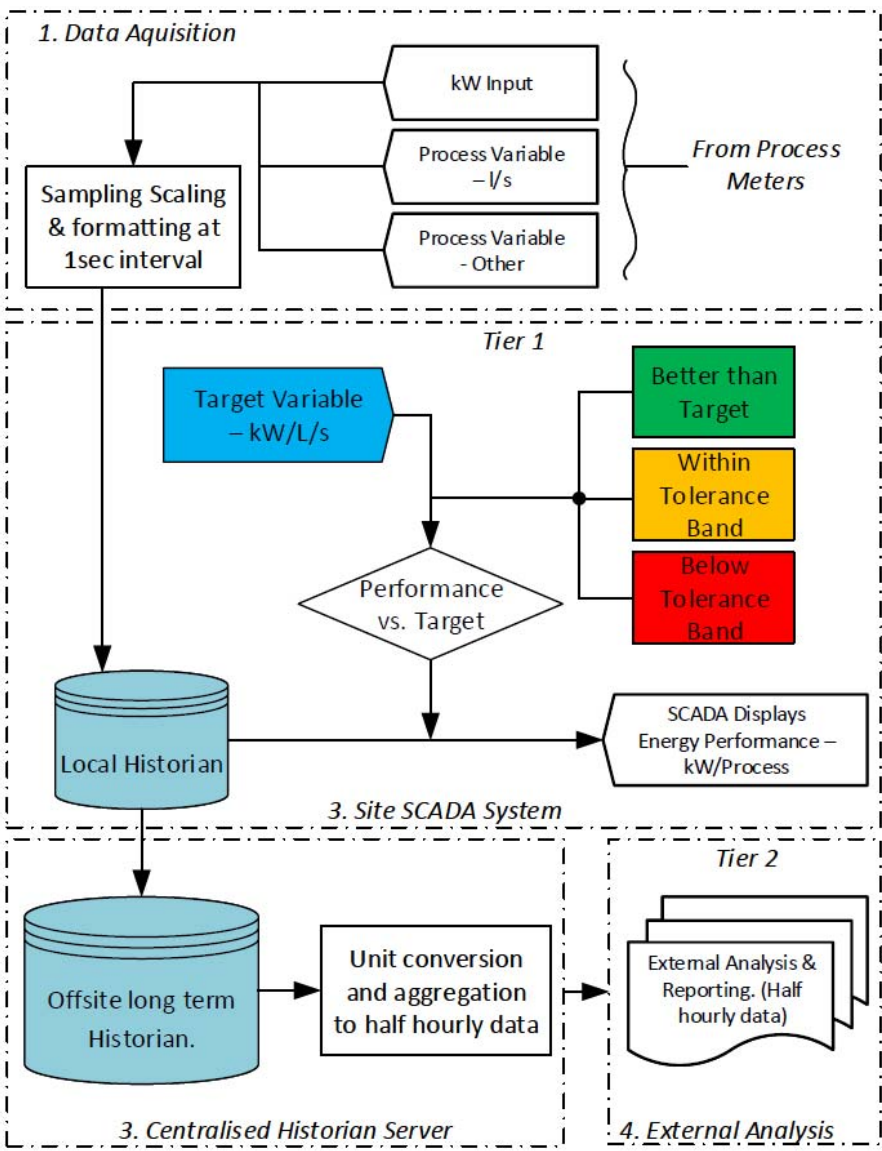

Fig. 8. Data path through the sub metering architecture

sites/asset's performance ranging from the previous hour to the previous 10 days, as it was believed this should augment their ability to quickly identify any potential short-term performance changes.

For the longer-term requirements, the performance data was necessary more for an external high level layer of analysis and reporting by the site managers and business users, such as specialist carbon and energy analysts. This longer-term data was migrated from the local site SCADA historians to the off-site corporate historians.

To facilitate analysis, it was imperative to include a date/time stamp with every recorded value; furthermore, this timestamp had to be assigned when the data was acquired from the sub-meter and not when the data was necessarily uploaded to the historian. This method was employed, so as to protect against potential data-propagation latencies within the system. Although the SCADA system is configured as a time master, with all child nodes below synchronising to it for their own times; there are occasions where these synchronisations fail, or the refresh rate is not of high enough resolution to protect against the latencies in the data time stamps.

Fig. 8 is an overview of how the data is transferred between platforms, with originating within the individual process meters, such as the Socomec Diris ${ }^{\mathrm{TM}}$ A40, and finishing up as an external analysis tool. Each stage is described below.

\section{A. Data acquisition}

The relevant process meters were interrogated at a one second interval rate. The raw data inputs, which when considering a new sub-meter installation are presented in the form of a discrete 4-20mA signal, were then scaled and formatted to an engineering unit range. E.g. 4-20mA equates to $0-100 \mathrm{~kW}$.

Scaling was the single biggest issue with the accuracy of the data when commissioning the meters. This was because the Thames Water estate comprises many PLC and SCADA variants, in which multiple scaling ranges exist. Different combinations of the varying PLC and SCADA software's resulted in a large number of possible scaling options which was where errors were made. For example; a Rockwell CompactLogix ${ }^{\mathrm{TM}}$ PLC analogue input card can scale the raw input into four different formats; Raw/Proportional, Engineering Units, Scaled for PID and Percentage Range. TABLE 1 shows how these 4 data types can be presented within a controller, and assumes that the physical signal is scaled 4-20mA correctly.

TABLE 1

PlC SCALING EXAMPLES

\begin{tabular}{|l|l|}
\hline Operating Range $\mathbf{= 4 - 2 0 m A}$ & Data Range \\
\hline Raw/Proportional & $-32,767$ to 32,767 \\
\hline Engineering Units & 3,200 to 21,000 \\
\hline Scaled for PID & 0 to 16383 \\
\hline Percentage Range & 0 to 10,000 \\
\hline
\end{tabular}

Further to this, the corporate SCADA systems used to historise and report on this data also differed from site to site. This added extra potential for errors within the scaling, and presented a major challenge to the project team in ensuring the data remained accurate from source to final destination.

In addition, resource skills/gaps at the time would occasionally impact this. There were incidents were one resource that was proficient with a certain type of PLC, was not as proficient with the particular site SCADA type. Therefore, there was a need for multiple resources to be involved in the commissioning of these signals, which led to some mistakes being made in the scaling throughout the data cycle. There was also the fact that the electrical commissioning of the sub-meter was completed separately to the SCADA commissioning and therefore any issues were not found until much later when signal accuracy could not be established.

\section{B. SCADA system}

The process data is analysed by the SCADA software and displayed on the local mimics. The energy performance is compared to target variables and alerts were set up to trigger when the actual performance moved out of range. Date and time stamps were added to the data, where it was stored in the local database historian.

Another issue encountered was that many of the SCADA applications across the Thames Water estate were obsolete, and running on end of life operating systems and hardware, much of which was no longer supported by the 
manufacturers. The extraction of the data for reporting purposes from these systems was at times not possible and was limited to pockets of sites where newer applications were utilised [11]. In this instance, a $3^{\text {rd }}$ party 'soft telemetry outstation' was installed on the server hardware, and used to collect the data via an Open Platform Communications (OPC) link.

\section{Centralised server}

The data from the local historian was then transferred to an offsite historian database. The data was reduced to samples based on 5 minute intervals. A facility within the offsite historian was used to aggregate the data to half hourly values.

\section{External analysis}

The data was then presentable such that it could be exported for external analysis or loaded into an SQL database in which queries could be run to perform high level analysis of the data.

\section{BenEFIts Following ENERGy DATA ACCESS}

Following the installation of 405 energy sub-meters, that were fitted and commissioned down the electrical distribution systems, and once verified as accurate provided granular data on individual feeders and pumps; gave all business users an enhanced visualisation of the site demands. This in turn enabled further optimisation of site performances. The optimisation primarily came from identifying faulty and/or failing assets. These were then (where process allowed) taken out of service or replaced. Also, where other process variables were already existing, such as flows or levels, these were correlated against the new power signals to give enhanced power against work done trends, which then provided an early warning system when devices started to fall below their expected output. This has led to a reduced local demand of $6.5 \mathrm{GWh} /$ year.

The improvement in available energy data also contributed to the further optimisation of 8 sewage aeration treatment systems, improving their controls; thus, achieving a further power reduction of $3.7 \mathrm{GWh} /$ year $[12,13]$.

\section{CONCLUSIONS}

The sub-metering project set out to achieve an enhanced understanding of where inefficiencies existed within a water company's electrical asset base, which it achieved across a cross section of devices in the estate. There has been significant improvement in the way in which these assets are owned and operated; including improved forecasting of where devices are beginning to fail, allowing for prioritisation of asset upgrade, or as a precautionary method, demoting the asset in its duties until such time as funding becomes available for replacement.

There has been a clear reduction in the use of electrical energy, with smarter asset utilisation. This has led to commercial savings, which has driven the business towards increasing sub-metering throughout the estate. However, it should be noted that, in some cases, the capital outlay required to achieve sub-metering on devices may not be the most efficient means of saving money, as the cost of installation far outweighs any payback return periods. If an MCC is on the roadmap for replacement within any current or following asset management period, then it will be more cost efficient to prioritise an alternative site for sub-metering installation, where greater benefits can be realised.

Once the complex electrical installation is complete and the $4-20 \mathrm{~mA}$ signals are presented to the PLC, there could be further project savings made by having a standardised approach in terms of data scaling and multi skilled resource that can complete the data transfer between all systems. This could be achieved via a standardised approach in terms of data scaling and presentation, as well as a framework of data block templates, which have embedded functions and are able to be adaptable across multiple SCADA platforms.

\section{ACKNOWLEDGEMENTS}

This paper is the result of a joint effort between Thames Water and Brunel University London. It is part of a research degree being completed by Alex Gray, which is focused on optimising the use of electrical energy within the waste water industry through improved utilization of process control and automation. The research is scheduled to complete by October 2018.

\section{REFERENCES}

[1] Kamilaris, A., et al., A literature survey on measuring energy usage for miscellaneous electric loads in offices and commercial buildings. Renewable and Sustainable Energy Reviews, 2014. 34: p. 536-550.

[2] Krishnanand, K.R., et al. Electrical submetering with repurposable applications for the built environment. in IECON 2016 - 42nd Annual Conference of the IEEE Industrial Electronics Society. 2016.

[3] Power, H. Haven Power signs $£ 520 \mathrm{~m}$ deal to provide Thames Water with 100\% renewable energy. 2015 [cited 2017 25th May]; Available from: http://www.havenpower.com/news/haven-power-signs-520mdeal-to-provide-thames-water-with-100-renewable-energy/2272.

[4] Gray, A.D., et al. Optimising the use of electrical energy within the waste water industry through improved utilization of process control and automation. in 2015 50th International Universities Power Engineering Conference (UPEC). 2015.

[5] OFWAT, Future water and sewerage charges 2010-15: Final Determinations OFWAT, Editor. 2009, OFWAT.

[6] Parliament, The Measuring Instruments (ActiveElectrical Energy Meters) Regulations 2006. 2006.

[7] UKGOV. MID approved gas and electricity meters. 2014 [cited 2017 25th May]; Available from: https:/www.gov.uk/guidance/midapproved-gas-and-electricity-meters.

[8] Tektronix, The Fundamentals of Three-Phase Power Measurements, T. Inc, Editor. 2013. p. 8.

[9] Group, S., DIRIS A40/A41 - Operating instructions, Socomec, Editor. 2013, Socomec SA. p. 84.

[10] Lindmark, J. and M. Håkansson. Smart Grid using $1<$ sup $>$ st $</$ sup $>$ generation AMR meters \&\#x2014; An operational view. in 22nd International Conference and Exhibition on Electricity Distribution (CIRED 2013). 2013.

[11] Gray, A.D.L., et al., A Standardised Modular Approach for Site SCADA Applications within a Water Utility. IEEE Access, 2017. PP(99): p. 1-1.

[12] Water, T. About Us - Asset Optimisation. 2014 1st February 2015]; Available from: www.thameswater.co.uk/about-us/10098.htm

[13] Water, T. Climate Change and Carbon Footprint. 2014 1st February 2015]; Available from: www.thameswater.co.uk/cr/Climatechange/Mitigatingclimatechange/M anagingourcarbonfootprint/index.html. 